Research Article

\title{
Exploration of Ziziphi Spinosae Semen in Treating Insomnia Based on Network Pharmacology Strategy
}

\author{
Gong Feipeng, ${ }^{1,2}$ Xie Luxin, ${ }^{1}$ Chen Beili, ${ }^{3}$ Yang Songhong $\left(\mathbb{D},{ }^{1}\right.$ Wu Wenting, ${ }^{1}$ Li Junmao, \\ Gong Qianfeng $\mathbb{D}^{1},{ }^{1}$ Zhong Lingyun $\mathbb{D}^{1},{ }^{1}$ and $W u$ Jianxiong $\mathbb{D D}^{1}$ \\ ${ }^{1}$ Jiangxi University of Chinese Medicine, Nanchang, China \\ ${ }^{2}$ Jiangxi Provincial People's Hospital Affiliated to Nanchang University, Nanchang, China \\ ${ }^{3}$ Tiantai County Food and Drug Testing Center, Taizhou, China
}

Correspondence should be addressed to Gong Qianfeng; gongqf2002@163.com, Zhong Lingyun; ly1638163@163.com, and Wu Jianxiong; 281105184@qq.com

Received 22 July 2021; Accepted 4 October 2021; Published 28 October 2021

Academic Editor: Junqing Huang

Copyright ( 2021 Gong Feipeng et al. This is an open access article distributed under the Creative Commons Attribution License, which permits unrestricted use, distribution, and reproduction in any medium, provided the original work is properly cited.

\begin{abstract}
Ziziphi Spinosae Semen (ZSS) is a common natural medicine used to treat insomnia, and to show clearly its method of action, we managed and did an in-depth discussion. Network pharmacology research is very suitable for the analysis of multiple components, multiple targets, and multiple pathways of Traditional Chinese Medicine (TCM). According to the relevant theory, we first carefully collected and screened the active ingredients in ZSS and received 11 active ingredients that may work. The targets going along with these active components were also strongly related to insomnia targets, 108 common genes were identified, and drugcompound-gene symbol-disease visualization network and protein-protein interaction network were constructed. Forty-eight core genes were identified by PPI analysis and subjected to GO functional analysis with KEGG pathway analysis. The results of GO analysis pointed that there were 998 gene ontology items for the treatment of insomnia, including terms of 892 biological processes, 47 cellular components, and 59 molecular functions. It mainly shows the coupling effect and transport mode of some proteins in the biological pathways of ZSS in the treatment of insomnia and explains the mechanism of action through the connection between the target and the cell biomembrane. KEGG enrichment analyzed 19 signaling pathways, which were collectively classified into seven categories. We have identified the potential pathways of ZSS against insomnia and obtained the regulatory relationship between core genes and pathways and know that the same target can be regulated by multiple components at the same time. The results of molecular docking also prove this conclusion. We sought to provide a new analytical approach to explore TCM treatments for diseases using network pharmacology analysis tools.
\end{abstract}

\section{Introduction}

Insomnia refers to the symptoms of unable to get commonly and regular sleep or difficulty falling asleep, insufficient sleep time, lack of deep sleep, or even sleepless nights. Sleep deprivation is a potential factor for a variety of risk sicknesses. General medicine-based treatments such as cognitive behavioral therapy for insomnia have limitations, and better alternatives have also been searched for [1]. There are many links between insomnia and mental health, of which anxiety and depression may have a bidirectional effect with insomnia, and the problem can no longer be ignored by people
[2]. Such people are prone to dizziness and brain swelling, generalized weakness, explosive temper, and irritability in the morning, which have been very harmful to the body for a long time in the past. With the fierce development of social competition, people's life rhythm has become faster. The prevalence and recurrence rates of insomnia remain high, and it has become one of the hot issues in the field of public health.

The effective drugs commonly used clinically are benzodiazepines, but they also have obvious side effects, even increasing the death rate of the elderly [3,4]. As an alternative to treating insomnia, Chinese medicine has achieved 
good therapeutic effects and has gradually attracted the attention of clinicians [5]. Ziziphi Spinosae Semen (ZSS) is the dried mature seed of Rhamnaceae plant Ziziphus jujuba Mill.var spinosa (Bunge) $\mathrm{Hu}$ ex $\mathrm{H}$. F. Chou; according to TCM theory, ZSS is a gentle temperament drug that can nourish the heart and benefit the liver, tranquilize the mind, converge sweat, and generate fluid [6]. ZSS is a relatively easy-to-obtain natural medicine. It is found in many Chinese medicine prescriptions for calming and tranquilizing the nerves, and it is most commonly used to treat insomnia [7].

Chinese herbal medicine has been used to treat diseases in China for thousands of years. Drugs regulate the immune system or nervous system by eliminating pathogenic factors and strengthening vital energy, so that various organs of the human body can operate normally and maintain the balance between the body and nature. Traditional Chinese herbs, on the other hand, are often multicomponent, multitarget, and multicenter of action. This paper also explains the treatment of insomnia by ZSS with the help of tools and analysis methods (Figure 1).

\section{Materials and Methods}

2.1. Screening of Active Compounds. To get the compounds in ZSS directly and effectively, we used the most commonly used specialized Traditional Chinese Medicine Systems Pharmacology Database and Analysis Platform (http://lsp. nwu.edu.cn/tcmsp.php); this platform relatively comprehensively covers the vast majority of TCM compounds' described information, while including the links between related targets and diseases [8, 9]. All ingredients about ZSS were first collected as candidates with the Chinese name "Suanzaoren" as the search term. Afterwards, we introduced these limiting parameters of oral bioavailability (OB) and drug-likeness (DL) according to the classical active ingredient screening rules [10], in which $O B$ refers to the percentage of unmodified drugs that enter the circulatory system after oral administration and can effectively represent the availability of drugs, and $\mathrm{OB} \geq 30 \%$ of compounds is considered as one of the screening rules for active ingredients [11]. While DL refers to the similarity of compounds to clinically used drugs, the greater DL value represents the possibility of compounds becoming drugs, and molecules with $\mathrm{DL} \geq 0.18$ are carefully thought about to have better pharmacological effects and are also one of the most commonly used active ingredient screening rules [12], so we started obeying this rule. In addition, as a drug often used by Chinese clinicians, the active substances of traditional Chinese medicine must meet the relevant provisions of the Chinese Pharmacopoeia when prescribing [13]. We also added the active ingredients specified in the Chinese Pharmacopoeia as active compounds.

2.2. Identification of Targets and Gene Symbols Associated with ZSS Compounds. The SMILES structural formulas of the compounds were obtained with the ZINC 15 database [14] and TCMSP Platform according to the CAS number, and the SMILES formulas were input into the SwissTargetPrediction
(STP; http://www.swisstargetprediction.ch) [15]. We use the information given to extract the protein targets of each ZSSactive compounds and simplify the obtained data to get gene symbols.

2.3. Acquisition of Insomnia Gene Targets. In order to ensure that the genes we obtain that are associated with the disease are more comprehensive, we used "Insomnia" as a keyword to search on these two reliable database platforms, respectively, from the GeneCards database (https://www. genecards.org/) and the Online Mendelian Inheritance in Man (OMIM) database (http://www.omim.org/). GeneCards database automatically integrates data from about 125 network-derived genes including genome, transcriptomics, and proteomics, with very powerful functions [16, 17]. The OMIM database can question almost any data on genetic diseases and provide linkage relationships known about causative genes $[18,19]$. After summarizing the obtained disease information, it is then screened in detail. Eventually, we obtained genes associated with insomnia.

2.4. Drug-Compound-Gene-Disease (D-C-G-D) Network Construction. We first obtained overlapping targets by crossing ZSS-related targets with insomnia-related targets by Venn diagram and then built a visual comprehensive network (D-C-G-D) based on the interaction between drugs (ZSS), compounds, gene symbols, and diseases (insomnia) by Cytoscape software (version 3.8.0) and made the corresponding schematic.

\subsection{Protein-Protein Interaction (PPI) Network Construction.} Discovering and annotating the interaction relationships of all functional targets in proteins enable system-level learning and understanding of the functions of proteins, and proteinprotein interaction data were obtained from the STRING database (https://string-db.org/). STRING is commonly used to filter and evaluate relevant data for functional genomics, setting the organism species to Homo sapiens (human) before retrieval [20] and then organizing the received data.

2.6. Gene Ontology (GO) and Kyoto Encyclopedia of Genes and Genomes (KEGG) Pathway Enrichment. Gene Ontology (GO) enrichment analyses was performed with R software (version 4.0.2), and the entries enriched were determined by the Bioconductor database (http://bioconductor.org/). Kyoto Encyclopedia of Genes and Genomes (KEGG) enrichment analyses were performed using ClueGO plug-in in Cytoscape [21].

2.7. Computational Validation of Compound-Target Interactions. As a feasible and modern verification method, molecular docking is a method for drug design through the characteristics of receptors and the interaction mode between receptors and drug molecules and is widely used in the field of drug binding to protein receptors [22]. We selected 


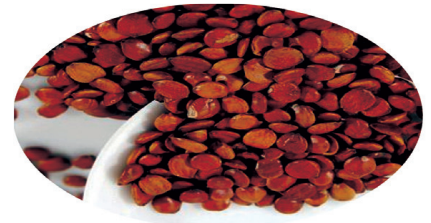

Ziziphi Spinosae Semen

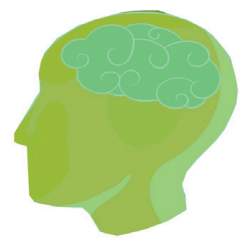

Insomnia
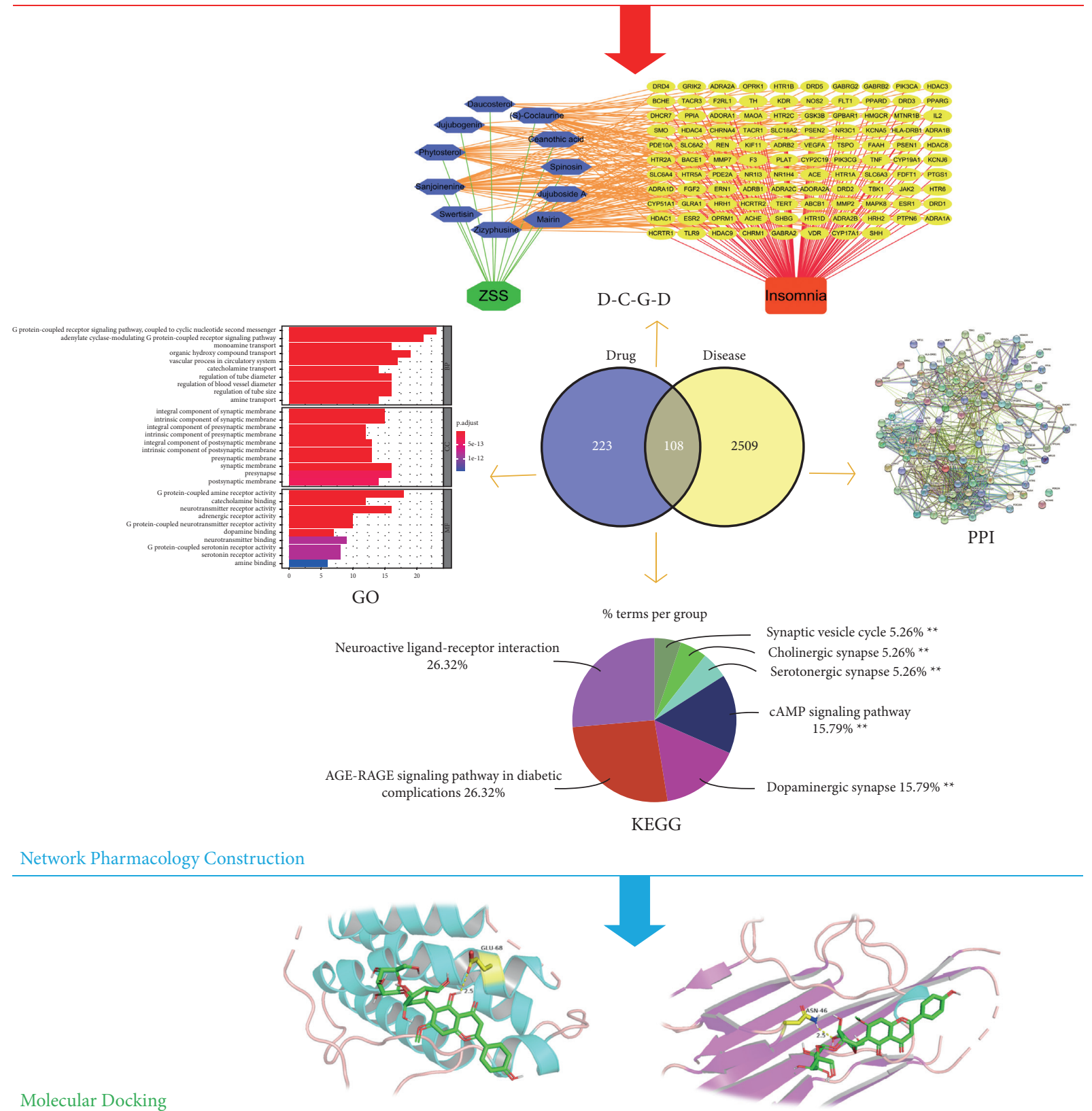

FIGURE 1: A comprehensive strategy diagram for the study of the mechanism of ZSS acting on insomnia.

three active ingredients for docking with their corresponding two targets, for a total of four component-target interactions to validate the prediction results. The protein crystal forms matching up to all targets were obtained from the RCSB Protein Data Bank (PDB) (https://www.rcsb.org). We chose the appropriate protein crystal overall principle to be structurally intact and stable and obtained X-ray crystal structures of TNF (TNF-alpha) and IL-2 (interleukin-2), which have a PDBID of 5 UUI and 1 M4B, respectively. In addition, to further confirm the reliability of the docking results, we also docked these two proteins with their positive inhibitors curcumin and upadacitinib. After processing 
targets and compounds using PyMOL software (version 1.3), docking work was performed using AutoDock Vina software (version 1.1.2), and the presentation files were processed by AutoDockTools.

\section{Results}

3.1. Screening of Active Compounds. We collated the collected data and compared the obtained component names, CAS numbers, and structures one by one to remove some inaccurate information. Nine active ingredients that met the above conditions were obtained by screening according to the previous parameters of $\mathrm{OB} \geq 30 \%$ and $\mathrm{DL} \geq 0.18$. In addition, two components, jujuboside $\mathrm{A}$ and spinosin, were included in the provisions of Chinese Pharmacopoeia 2020. Studies have shown that saponins and flavonoids are the two most important active components in ZSS $[23,24]$, the most important of which are jujuboside A and spinosin. Jujuboside A can inhibit cancer cell growth through various mechanisms such as cell cycle arrest, proliferation inhibition, stem cell inhibition, and promotion of aging and can contaminate antitoxins [25, 26], and our analysis results show that there are many intersections of targets associated with it and insomnia targets. Spinosin exerts neuroprotective effects by inhibiting oxidative damage and has a significant antidepressant effect, and there is a great association between spinosin and insomnia [27, 28]. Although the kinetic values of these two components are relatively low, they have very excellent biological activity and are closely related to our study. Finally, all eligible compounds were combined and we obtained 11 fractions as final active compounds (Table 1).

3.2. Identification of Targets and Gene Symbols Associated with ZSS Compounds. After collecting related proteins from the STP database and simplifying (probability $>0$ ), we obtained 11 components in the ZSS and 331 known target symbols associated with them (Supplementary Table S1).

3.3. Acquisition of Known Therapeutic Gene Targets for Insomnia. We merged all target data obtained from the GeneCards and OMIM databases for insomnia-related targets and removed duplicated genes from them, collecting 2617 known insomnia therapeutic targets (Supplementary Table S2).

3.4. D-C-G-D Network Construction. According to Venn diagram (Figure 2(a)), we can visually see the cross genes common to drugs and diseases, of which 331 drug gene symbols and 2617 disease gene symbols have 108 overlaps. The D-C-G-D network (Figure 2(b)) also clearly shows how ZSS treats insomnia by acting between components and targets, and the details of the D-C-G-D network are shown in Supplementary Table S3.

3.5. PPI Network Construction. According to the results received by STRING platform, we got the action relationship map among 108 overlapping genes (Figure 3(a)), including two unconnected free genes, which may be important targets of ZSS to treat insomnia. After removing the free genes, we calculated the topological index node degree of 106 genes, which largely points to the degree of association of this gene with other genes. The higher node degree indicates that this gene may be more important, and the details of the specific gene name and its corresponding node degree are given in Supplementary Table S4. In addition, we calculated the mean value of 106 overlapping gene degrees to be 15.05, selected all genes with value greater than the mean value [34], and generated 48 core targets of relatively more important networks with other gene interactions (Figure 3(b)). Moreover, to better highlight the importance of key targets, after processing these related targets using Cytoscape software, it displayed 48 genes with higher values in the inner loop of Figure 3(c). The deeper the blue color and the larger the nodes, the higher the node degree; the line between them represented the interaction. These core targets may be the key genes of ZSS in the treatment of insomnia.

3.6. GO and KEGG Pathway Enrichment. GO enrichment analysis $(p<0.01)$ of 48 core target genes was classified and enriched according to three modules: biological process (BP), cellular component (CC), and molecular function (MF), with 998 GO terms enriched, of which the BP term accounted for a relatively high proportion, with $892 \mathrm{GO}$ terms, mainly showing the coupling role and transport mode of some proteins in biological pathways, such as G proteincoupled receptor signaling pathway, coupled to cyclic nucleotide second messenger (GO:0007187), adenylate cyclasemodulating $G$ protein-coupled receptor signaling pathway (GO:0007188), monoamine transport (GO:0015844), and organic hydroxy compound transport (GO:0015850). CC is rich in 47 GO terms and analyzes that these core genes are closely related to those cell biofilms, mainly involving a variety of synaptic membranes, such as integral component of synaptic membrane (GO:0099699), intrinsic component of synaptic membrane (GO:0099240), and integral component of presynaptic membrane (GO:0099056). Similarly, we enriched $59 \mathrm{GO}$ terms for MF, and in MF analysis, we could understand which receptor activities the core genes would affect and the forms of partial protein and target binding, mainly $\mathrm{G}$ protein-coupled amine receptor activity (GO:0008227), neurotransmitter receptor activity (GO: 0030594), and catecholamine binding (GO:1901338). To present the GO enrichment results more directly, we based on $p$. Adjust intercepts for the top 10 terms from small to large for an abbreviated presentation (Figure 4), respectively, and the detailed results of the specific GO item analysis are given in Supplementary Table S5.

KEGG pathway enrichment analysis can help us further figure out the potential pathways of ZSS against insomnia; after all core genes are entered and after applying $p$ value significance selection criteria, 44 genes from all clusters are systematically selected and 19 representative pathways are selected $(p<0.01 p<0.01)$, as shown in Figure 5(a), and these pathways are divided into 7 categories by signaling pathway functional clustering analysis, of which neuroactive ligand- 
TABLE 1: Final active compounds selected as the details of ZSS in this study.

\begin{tabular}{lccccccc}
\hline No. & Molecule name & MF & MW & OB (\%) & DL & CAS number & References \\
\hline 1 & Ceanothic acid & C30H46O5 & 486.76 & 33.41 & 0.77 & $21302-79-4$ & {$[29]$} \\
2 & (S)-coclaurine & C17H19NO3 & 285.37 & 42.35 & 0.24 & $486-39-5$ & {$[30]$} \\
3 & Daucosterol & C35H60O6 & 576.80 & 36.91 & 0.75 & {$[31]$} \\
4 & Jujubogenin & C30H48O4 & 472.78 & 34.96 & 0.62 & $54815-36-0$ & {$[32]$} \\
5 & Phytosterol & C29H50O & 414.79 & 36.91 & 0.75 & $949109-75-5$ & {$[31]$} \\
6 & Sanjoinenine & C29H35N3O4 & 489.67 & 67.27 & 0.79 & $107446-80-0$ & {$[31]$} \\
7 & Swertisin & C22H22O10 & 446.44 & 31.83 & 0.75 & $6991-10-2$ & {$[33]$} \\
8 & Zizyphusine & C20H24NO4 & 342.45 & 41.52 & 0.55 & $107446-79-7$ & {$[29]$} \\
9 & Mairin & C30H48O3 & 456.78 & 55.37 & 0.78 & $472-15-1$ & {$[31]$} \\
10 & Jujuboside A & C58H94O26 & 1207.52 & 8.03 & 0.02 & $55466-04-1$ & {$[30]$} \\
11 & Spinosin & C28H32O15 & 608.60 & 6.31 & 0.72 & $72063-39-9$ & {$[33]$} \\
\hline
\end{tabular}

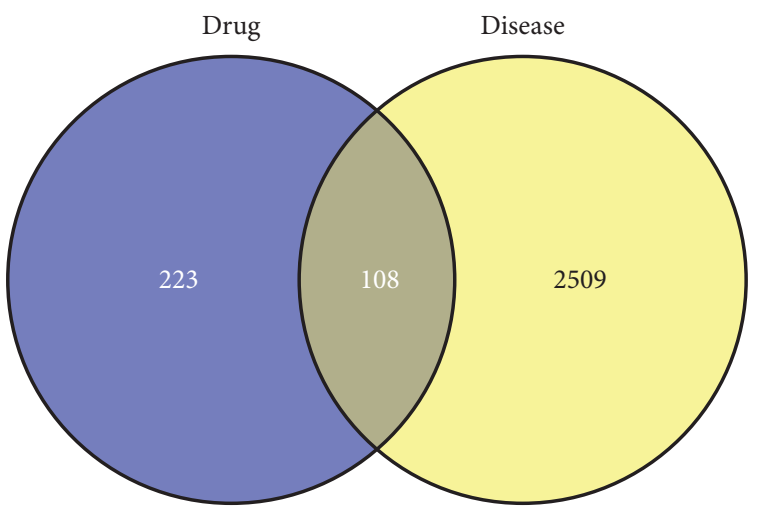

(a)

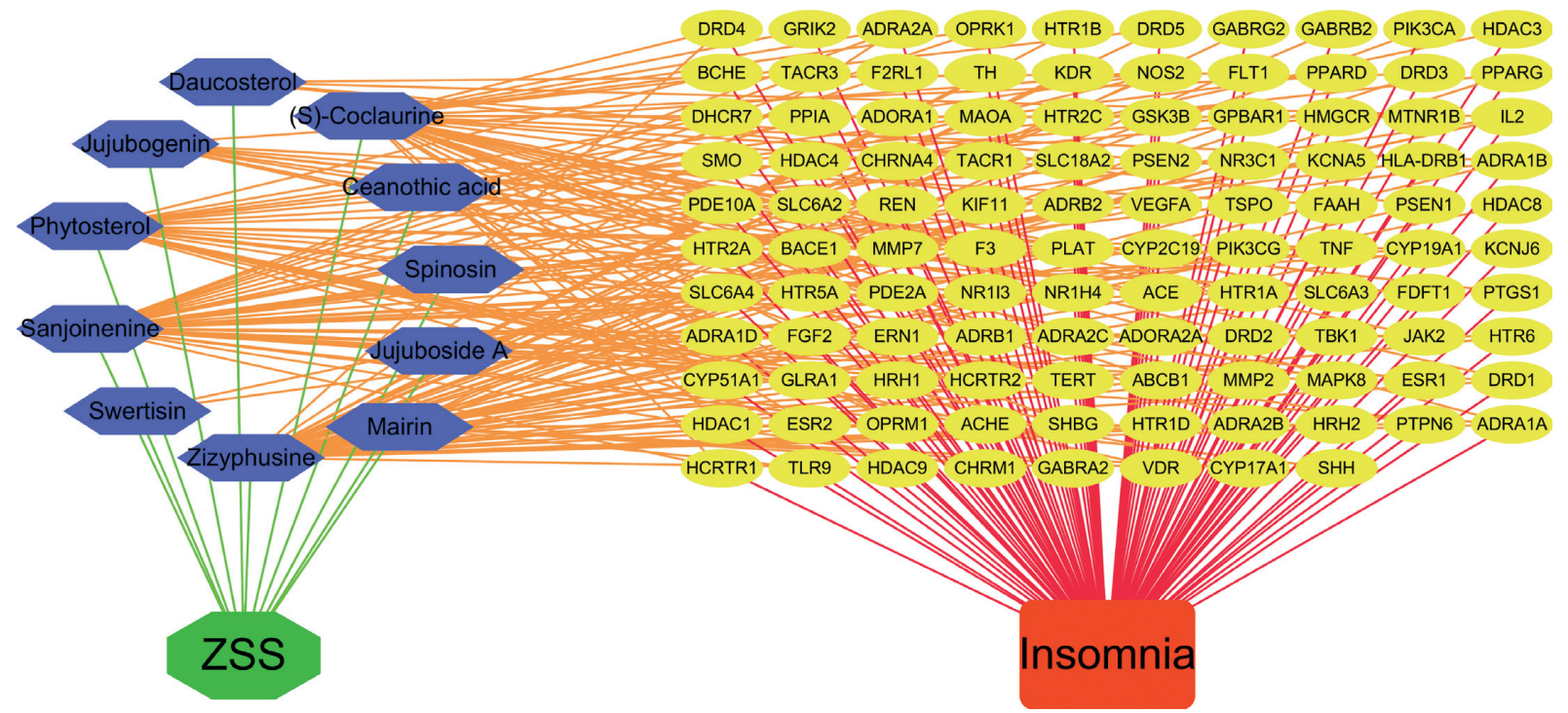

(b)

FIGURE 2: (a) Venn diagram of related targets of ZSS and insomnia. (b) D-C-G-D network. Green and red nodes indicate ZSS and insomnia, respectively. 11 blue nodes represent active ingredients in ZSS, 108 yellow nodes represent overlapping gene symbols between disease and drug, with edges indicating that nodes can interact, red edges indicate the action of insomnia with genes, green edges indicate the interaction of ZSS with active ingredients, and orange edges indicate the interaction of active ingredients with genes.

receptor interaction (KEGG:04080), AGE-RAGE signaling pathway in diabetic complications (KEGG:04933), dopaminergic synapse (KEGG:04728), cAMP signaling pathway (KEGG:04024), synaptic vesicle cycle (KEGG:04721), serotonergic synapse (KEGG:04726), and cholinergic synapse (KEGG: 04725) each represent a class of biological pathways (Figure 5(b)). Some of these pathways have been shown to be highly connected with insomnia, such as key 


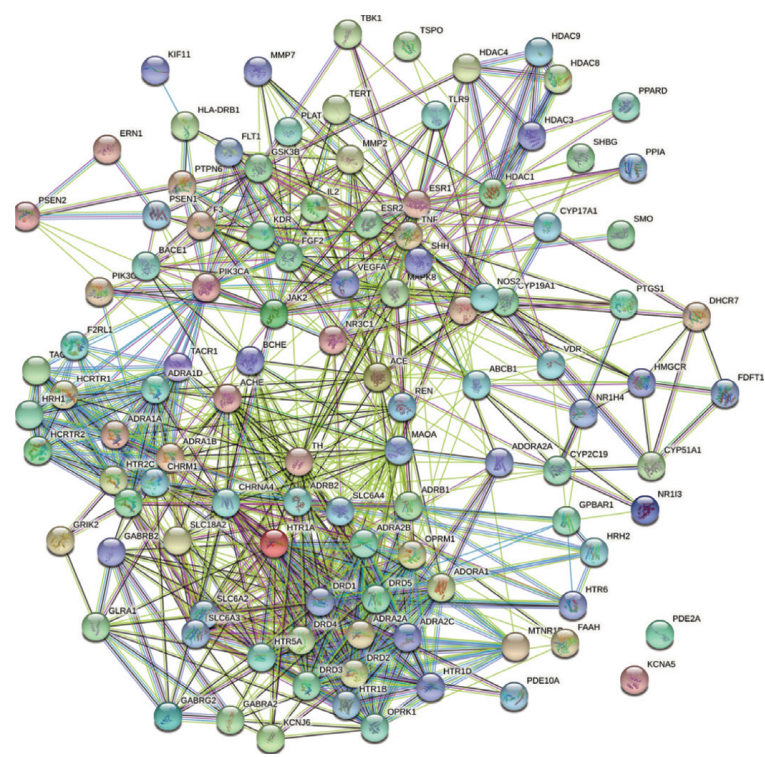

(a)

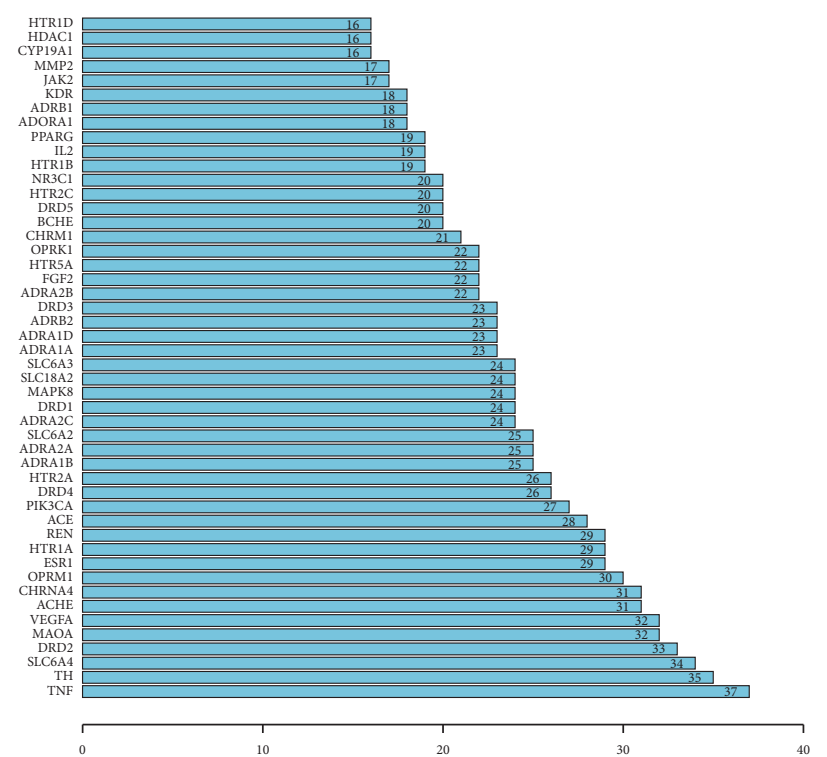

(b)

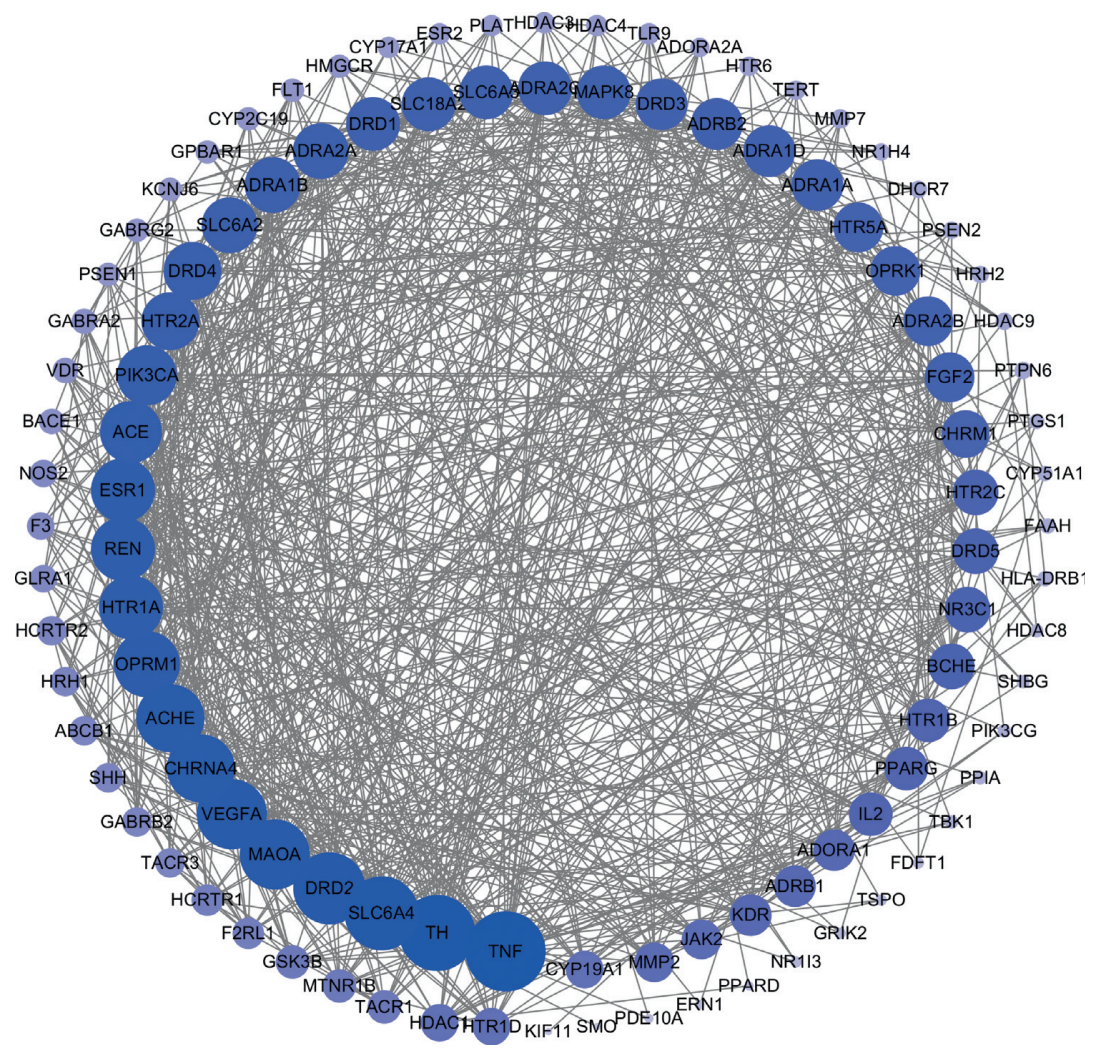

(c)

FIgure 3: (a) The PPI network obtained from the STRING database platform. (b) The core gene degree barplot diagram. (c) The PPI network arranged according to degree value.

protein pathways that upregulate neuroactive ligand-receptor interaction signaling that can significantly improve insomnia [35], and some pharmacological treatments for psychiatric disorders also use selective blockade of presynaptic dopamine receptors in the frontal cortex, enhancing dopaminergic transmission, which is consistent with the results of our analysis of modulation of the dopaminergic synapse signaling pathway [36]. All details of the KEGG pathway enrichment analysis are described in Supplementary Table S6.

3.7. Computational Validation of Ingredient-Target Interactions. Based on the above prediction and analysis, we selected three classical components, swertisin, jujuboside A, 

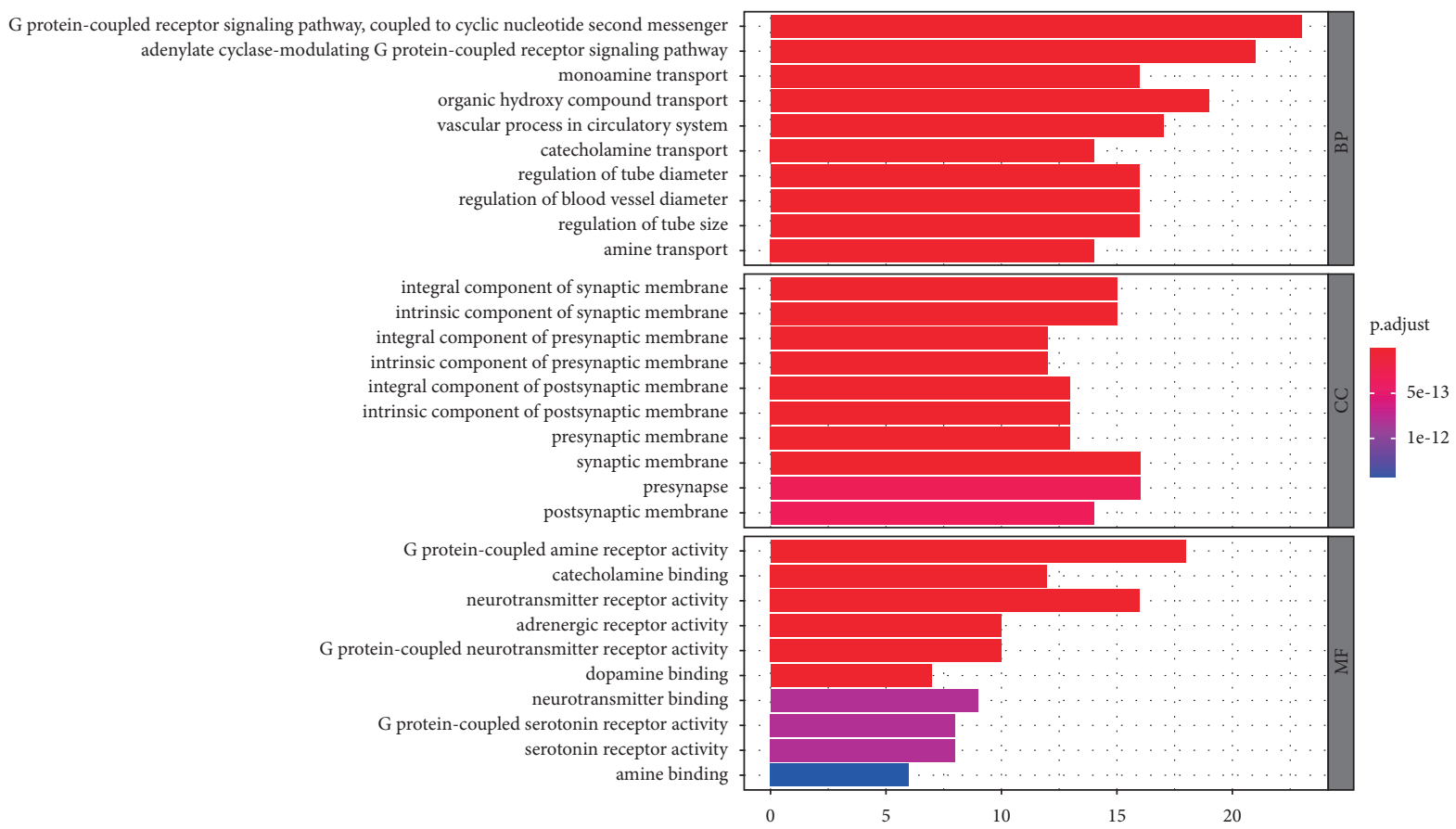

FIGURE 4: Bar graph of GO function enrichment of overlapping targets. The $Y$-axis represents GO terms. The $X$-axis indicates the number of genes enriched in this pathway. The redder the color, the smaller the p.adjust value; it also indicates the reliability and importance. The bluer the color, the greater the p.adjust value.

and spinosin, in ZSS for molecular docking with two important proteins, TNF and IL-2, to validate our analysis results. According to the docking results, we know that the binding energy of swertisin to TNF is $-6.4 \mathrm{kcal} / \mathrm{mol}$, the binding energy of jujuboside A to IL- 2 is $-7.8 \mathrm{kcal} / \mathrm{mol}$, and the binding energy of spinosin to TNF and IL-2 is -6.9 and $-5.9 \mathrm{kcal} / \mathrm{mol}$, respectively. The lower binding energy between molecules represents that the stronger force binding energy between them $(<0 \mathrm{kcal} / \mathrm{mol})$ is carefully thought about being conducive to the binding reaction, which shows that our analysis is accurate and reliable, and the compounds can better act on the related proteins. The binding energy of IL-2 and its inhibitor curcumin is $-6.5 \mathrm{kcal} / \mathrm{mol}$, and the binding energy of TNF and the inhibitor upadacitinib is $-6.4 \mathrm{kcal} / \mathrm{mol}$. The compound and the inhibitor are docked into the same pocket of the protein, which means our results are more reliable. Figure 6 shows the specific situation of their combination, and the molecule is represented in a ballstick model of atoms $\mathrm{C}, \mathrm{O}$, and $\mathrm{N}$ in green, red, and blue, respectively. Hydrogen bonds are indicated by dashed lines, and the numbers above represent distances in angstroms (A). Among them, two amino acid residues in IL-2, GLN-57 and GLU-68, form multiple binding locations with jujuboside A (Figure 6(a)), while GLU-68 also binds to spinosin through hydrogen bonding (Figure 6(c)). Three amino acid residues in TNF, GLN-47, LYS-90, and GLU-135-48, form multiple hydrogen bonds with swertisin (Figure 6(b)), and another amino acid residue, ASN-46, forms a hydrogen bond with spinosin (Figure 6(d)). It linked the positive inhibitor curcumin to the two amino acids, GLY-27 and ARG-83, on IL-2 (Figure 6(e)), and upadacitinib forms two hydrogen bonds with the amino acid ILE-136 on TNF
(Figure 6(f)). Our analysis shows that ZSS can effectively act on multiple targets such as TNF and IL-2 simultaneously through various components such as swertisin, jujuboside A, and spinosin, which makes the drug molecules affect the protein, and this achieves the effect of treating insomnia.

\section{Discussion}

With the acceleration of people's modern life rhythm, the number of insomnia population showed an increasing trend. Despite developed medical conditions and increasingly diverse means of treating psychiatric disorders, the problem of insomnia still cannot be effectively treated [37, 38]. Monomeric drugs have opened a channel of remission for some patients with neurological diseases, but it is undeniable that the effects of drug resistance and other side effects are not negligible [39, 40]. Most herbs originate from nature with relatively few side effects and adverse effects. ZSS is a pure natural traditional Chinese medicine that has a significant therapeutic effect on insomnia [41, 42], but its application and development have some limitations because of the complex mechanism of action of multiple components and multiple targets of traditional Chinese medicine. In this study, we used network pharmacology theory and related tools to select the active components and related targets of ZSS and linked them with insomnia-related targets to construct a D-C-G-D visual network, which more intuitively showed the specific relationship between drug components and diseases through 108 consensus genes. The results of PPI analysis showed complex interactions between overlapping genes, and 48 core targets were further screened according to the average node degree between them, such as TNF with a 


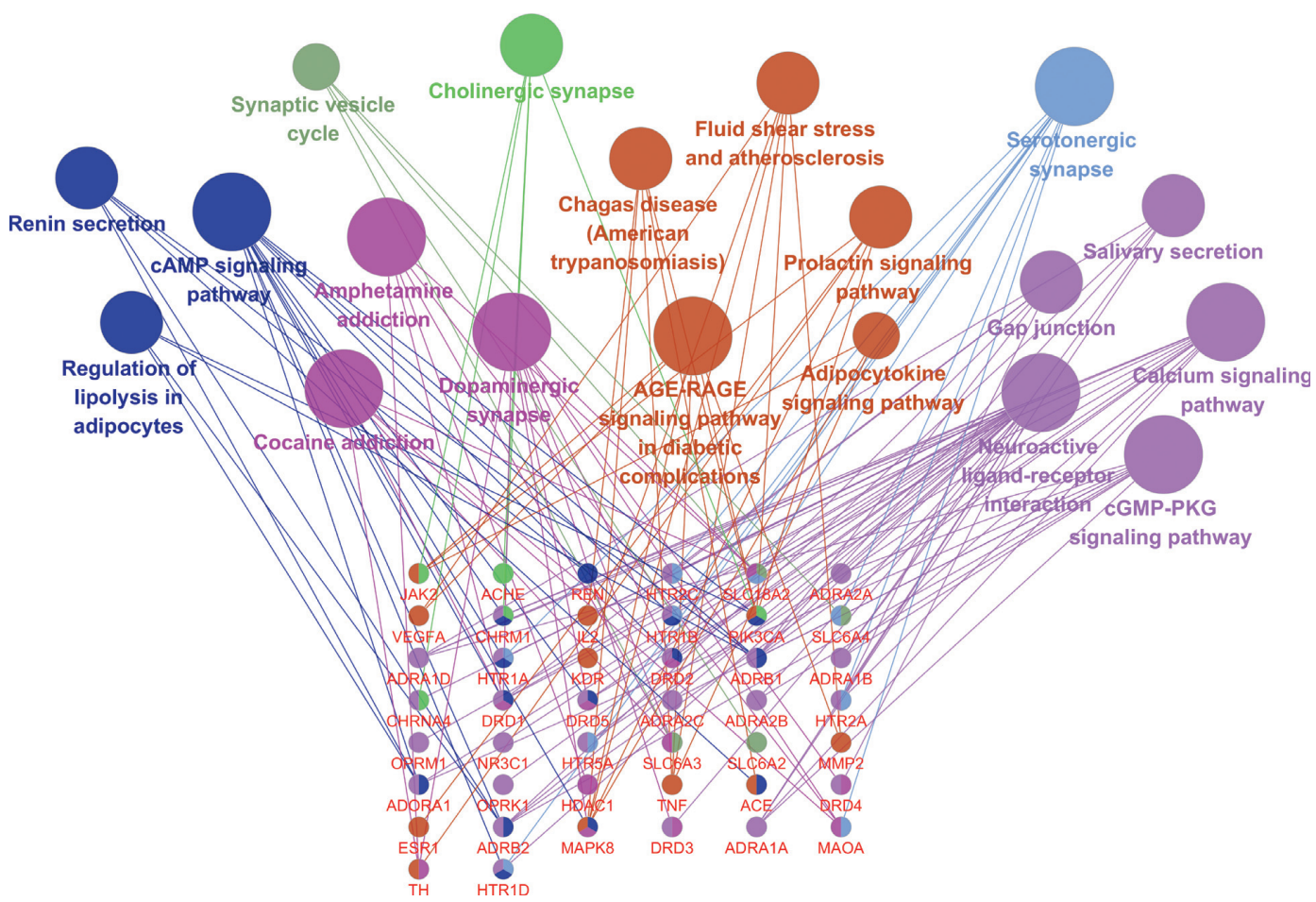

(a)

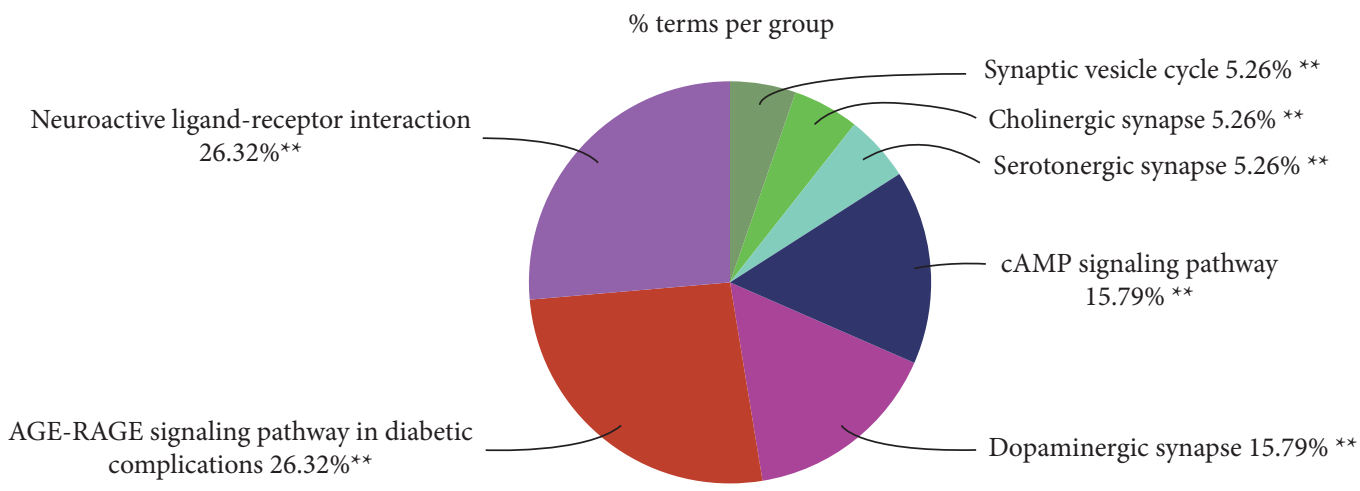

(b)

FIgURE 5: (a) Correlation diagram between core targets and KEGG pathway. The smaller the term p.adjust, the larger the node. Nodes with different colors represent different pathway classifications, and the line between pathway and gene represents the interaction between them. (b) Representative classification of pathways and percentage.

high node degree, meaning that one key to ZSS treatment of insomnia may be the inhibition of inflammation in vivo [43], which also made our subsequent analysis more prominent.

GO functional analysis makes a simple annotation of gene products, BP enrichment results in the coupling effect and transport mode of proteins in biological pathways, for example, $G$ protein-coupled receptors can mediate the normal function of the brain, of which $\mathrm{H} 3$ and $\mathrm{H} 4$ receptors are high-affinity receptors in the brain and immune system, respectively, and $\mathrm{H} 3$ receptors can regulate histamine and neurotransmitters, which control sleep quality [44]. The results of CC analysis show that intersection proteins or their products take part in the cellular environment, and active biofilm, such as a variety of protrusion membranes, can be utilized as tools to transmit synaptic currents in hypothalamic neurons, triggering arousal and regulating energy metabolism [45]. By MF analysis, we can determine that some protein receptor activities are regulated by drugs, such as neurotransmitter receptor, which is closely related to sleep, of which $\gamma$-amino butyric acid receptor plays an important role in controlling different vigilance states in the human body [46]. KEGG pathway analysis effectively helps us to associate genomes with cellular, species, and ecosystem functions. According to the results of our analysis, neuroactive ligand-receptor interaction, cAMP signaling pathway, and one of the key pathways to treat insomnia in ZSS, in the pathway, multiple targets such as ADRA1A, ADRA1B, and ADRA1D can be simultaneously regulated by components such as (S)-cointeraction, sanjoinenine, and zizyphusine, and upregulation of neuroactive ligand-receptor interaction 


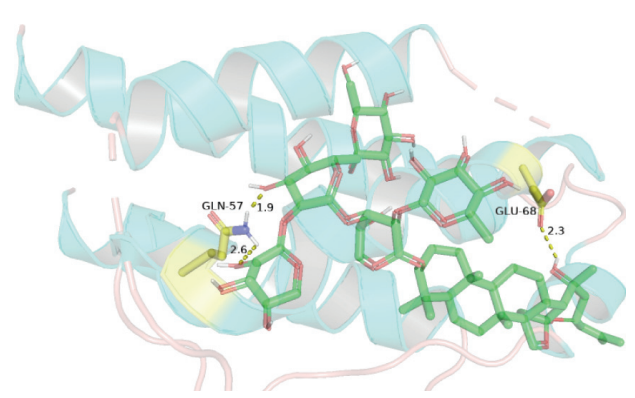

(a)

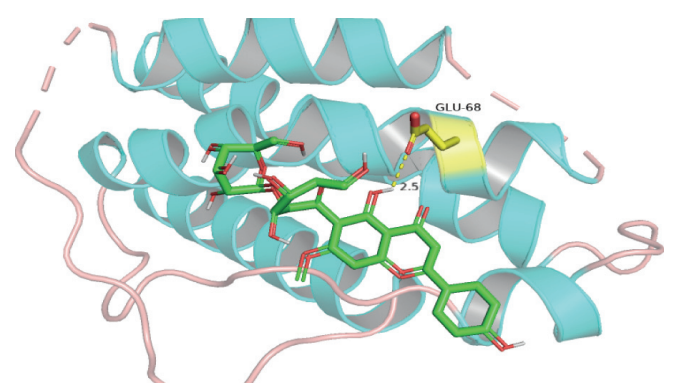

(c)

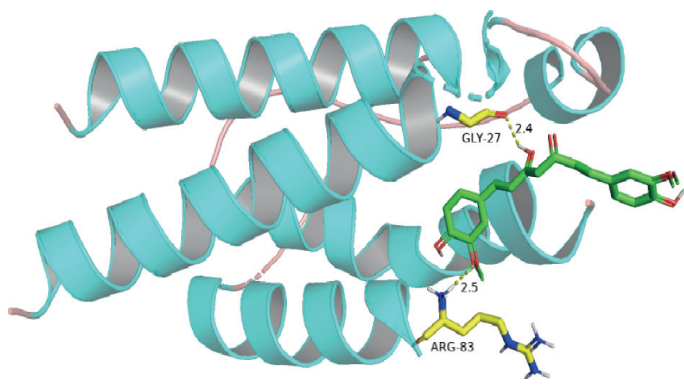

(e)

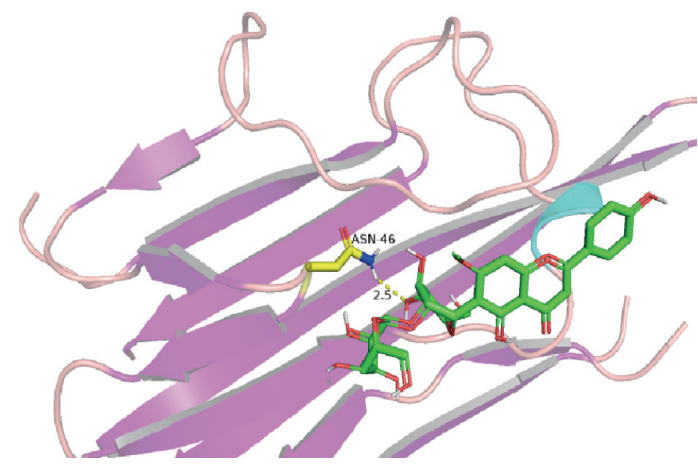

(b)

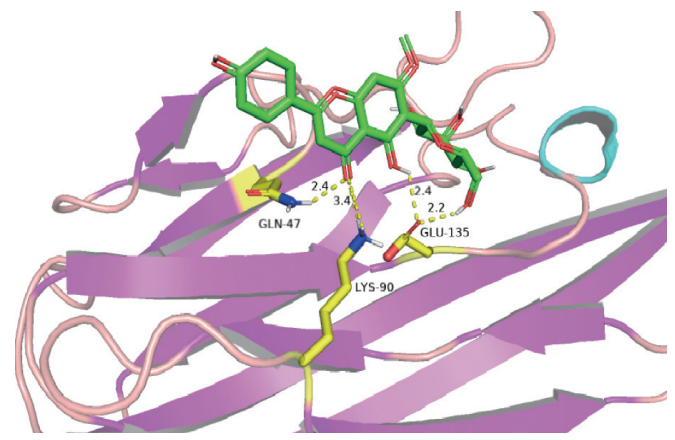

(d)

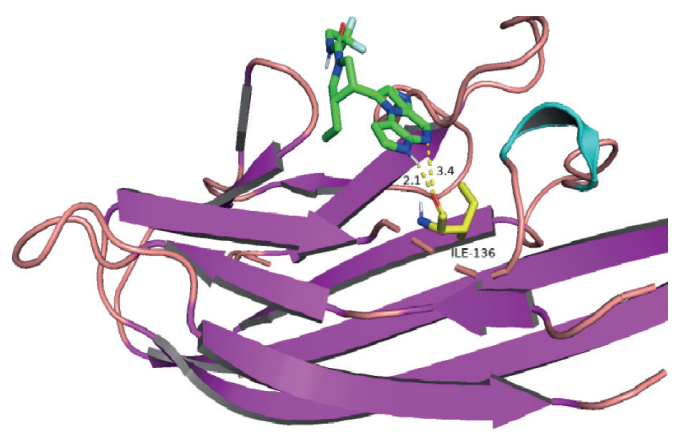

(f)

Figure 6: Selected compounds' interactions with the targets. (a) Jujuboside A with IL-2, (b) swertisin with TNF, (c) spinosin with IL-2, (d) spinosin with TNF, (e) curcumin with IL-2, and (f) upadacitinib with TNF. The molecule is represented in a ball-stick model with atoms $\mathrm{C}, \mathrm{O}$, and $\mathrm{N}$ in green, red, and blue, respectively. Hydrogen bonds are indicated by dashed lines, and the numbers above represent distances in angstroms $(\AA)$.

has also been an active process for the treatment of insomnia [35]. Besides identifying the specific pathways involved, cluster analysis of these pathways is also unique to our study, such as the cAMP signaling pathway, renin secretion, and dopaminergic synapse; these three pathways function similarly or are more closely linked during ZSS treatment of insomnia. Some protein kinases in the cAMP signaling pathway phosphorylate some specific proteins associated with insomnia, controlling the molecular and intracellular signaling of sleep and wake states [47]. Renin secretion can affect vital active states, and increased activity of the reninangiotensin system in the brain elevates fluid intake, blood pressure, and resting metabolic rate renin secretion [48]. In addition, antipsychotics may improve psychomotor and routine by releasing dopamine in the prefrontal cortex, which may also involve the dopaminergic synapse signaling pathway [36]. Classification analysis of signaling pathways reveals population differences between different signaling pathways when specific drugs are used to treat diseases. Based on the prediction results, we finally performed in silico molecular docking validation. The tight binding of a variety of small molecule compounds and amino acids on proteins preliminarily showed that our analysis was reliable, and it was also to affirm the feasibility of traditional Chinese medicine, an active compound in ZSS, in the treatment of insomnia.

Complex diseases often have many symptoms, and sometimes, the use of monomeric components is less effective to treat complex diseases and has significant side effects. TCM has been used to treat diseases for thousands of years, with significant results. Meanwhile, the special philosophical ideas of Chinese medicine and the very complex 
composition of TCM limit its further development, and the rise of network pharmacology brings another idea for the study of TCM. By constructing a drug-target-disease network, higher levels of information can be obtained by calculating the mechanism of simultaneous action of multiple components, multiple targets, and multiple pathways, and then using the construction of visual network charts can enable researchers to quickly and clearly know the mechanism of complex components in the treatment of diseases, which is a very practical new research tool. All of our work is dedicated to establishing a new analytical method with the help of network pharmacology, and besides exploring ZSS for the treatment of insomnia, we also hope to apply it in more traditional Chinese medicine research.

\section{Data Availability}

In this study, the sources are indicated in the text when the third-party data that may be involved are cited, and all datasets presented in this study are included within the article/supplementary materials and can be open to the public.

\section{Additional Points}

Contribution to the Field. Ziziphi Spinosae Semen (ZSS) has a unique effect in the field of insomnia and comforting the nerves, and the curative effect is remarkable. ZSS appears in many Chinese doctors' prescriptions for treating insomnia. It is known as the sleeping fruit of the East. However, at present, there are very few studies on how it works in the human body. With the help of network pharmacology, we conducted an association analysis on the important proteins of jujube seed in the treatment of insomnia for the first time and carefully studied the important biological pathways and cell functions that may be involved. Our work effectively makes the theoretical research of traditional Chinese medicine more closely integrated with the actual clinical application.

\section{Disclosure}

No human studies are presented in this article. No potentially identifiable human image or data are presented in this study.

\section{Conflicts of Interest}

The authors declare that the research was conducted in the absence of any commercial or financial relationships that could be construed as potential conflicts of interest.

\section{Authors' Contributions}

Gong Feipeng, Xie Luxin, and Chen Beili contributed equally to this work and are regarded as co-first authors.

\section{Acknowledgments}

This work was supported by the National Natural Science Foundation of China (81960716) and the National Key
Research and Development Plan (2018YFC1707206 and 2017YFC1702900). The authors are thankful to Jiangxi University of Chinese Medicine for the help in conducting this study.

\section{Supplementary Materials}

Table S1: detailed information of related targets and gene symbols of compounds in ZSS. Table S2: the known therapeutic gene targets for insomnia. Table S3: detailed information about the D-C-G-D network. Table S4: overlapping gene symbols and corresponding degree values between disease and drug. Table S5: details of GO enrichment analyses. Table S6: details of KEGG pathway enrichment analyses. (Supplementary Materials)

\section{References}

[1] F. Perini, K. F. Wong, J. Lin et al., "Mindfulness-based therapy for insomnia for older adults with sleep difficulties: a randomized clinical trial," Psychological Medicine, pp. 1-11, 2021.

[2] R. Stott, J. Pimm, R. Emsley, C. B. Miller, and C. A. Espie, "Does adjunctive digital CBT for insomnia improve clinical outcomes in an improving access to psychological therapies service?" Behaviour Research and Therapy, vol. 144, Article ID 103922, 2021.

[3] B. X. Lou and M. Oks, "Insomnia," Clinics in Geriatric Medicine, vol. 37, no. 3, pp. 401-415, 2021.

[4] M. Markota, T. A. Rummans, J. M. Bostwick, and M. I. Lapid, "Benzodiazepine use in older adults: dangers, management, and alternative therapies," Mayo Clinic Proceedings, vol. 91, no. 11, pp. 1632-1639, 2016.

[5] F. Li, X. Wang, Z. Song et al., "Efficacy and safety of traditional Chinese medicine yangxin anshen therapy for insomnia," Medicine, vol. 98, no. 37, Article ID e16945, 2019.

[6] PRC PCO, Pharmacopoeia of the People's Republic of China, China Medical Science Press, Beijing, China, 2020.

[7] X. Ni, J. L. Shergis, A. L. Zhang et al., "Traditional use of Chinese herbal medicine for insomnia and priorities setting of future clinical research," Journal of Alternative \& Complementary Medicine, vol. 25, no. 1, pp. 8-15, 2019.

[8] L. Zhou, W. Gu, F. Kui et al., "The mechanism and candidate compounds of aged citrus peel () preventing chronic obstructive pulmonary disease and its progression to lung cancer," Food \& Nutrition Research, vol. 65, 2021.

[9] H. Hu, K. Wang, L. Wang et al., "He-Jie-Shen-Shi decoction as an adjuvant therapy on severe coronavirus disease 2019: a retrospective cohort and potential mechanistic study," Frontiers in Pharmacology, vol. 12, Article ID 700498, 2021.

[10] Y. Chen, F. Bi, and Z. Sun, "A network pharmacology approach to determine the underlying mechanisms of action of Yishen Tongluo formula for the treatment of oligoasthenozoospermia," PLoS One, vol. 16, no. 6, Article ID e0252906, 2021.

[11] T.-C. Hung, N. Zhao, C. Huang et al., "Exploring the mechanism of PingTang No.5 capsule on nonalcoholic fatty liver disease through network pharmacology and experimental validation," Biomedicine \& Pharmacotherapy, vol. 138, Article ID 111408, 2021.

[12] L. Hu, Y. Chen, T. Chen, D. Huang, S. Li, and S. Cui, "A systematic study of mechanism of sargentodoxa cuneata and patrinia scabiosifolia against pelvic inflammatory disease with dampness-heat stasis syndrome via network pharmacology 
approach," Frontiers in Pharmacology, vol. 11, Article ID 582520, 2020.

[13] D. Wang, Q. Li, R. Liu et al., "Quality control of Semen Ziziphi Spinosae standard decoction based on determination of multi-components using TOF-MS/MS and UPLC-PDA technology," Journal of pharmaceutical analysis, vol. 9, no. 6, pp. 406-413, 2019.

[14] H. Gao, Y. Jiang, J. Zhan, and Y. Sun, "Pharmacophore-based drug design of $\mathrm{AChE}$ and $\mathrm{BChE}$ dual inhibitors as potential anti-Alzheimer's disease agents," Bioorganic Chemistry, vol. 114, Article ID 105149, 2021.

[15] X. Lu, G. Yan, M. Dawood et al., “A novel moniliformin derivative as pan-inhibitor of histone deacetylases triggering apoptosis of leukemia cells," Biochemical Pharmacology, Article ID 114677, 2021.

[16] J. Zhang, Y. Zhou, and Z. Ma, “Tripteryguim wilfordiiMultitarget mechanism of Hook for treatment of ankylosing spondylitis based on network pharmacology and molecular docking," Annals of Medicine, vol. 53, no. 1, pp. 1090-1098, 2021.

[17] Y. Shao, H. Jia, L. Huang et al., "An original ferroptosis-related gene signature effectively predicts the prognosis and clinical status for colorectal cancer patients," Frontiers in oncology, vol. 11, Article ID 711776, 2021.

[18] D. Shi, M. Motamed, A. Mejía-Benítez et al., "Genetic syndromes with diabetes: a systematic review," Obesity Reviews: An Official Journal of the International Association for the Study of Obesity, vol. 22, no. 9, Article ID e13303, 2021.

[19] Z. Yue, L. Li, H. Fu et al., "Effect of dapagliflozin on diabetic patients with cardiovascular disease via MAPK signalling pathway," Journal of Cellular and Molecular Medicine, vol. 25, no. 15, pp. 7500-7512, 2021.

[20] J. Wang, Y. Wu, M. N. Uddin, R. Chen, and J.-P. Hao, "Identification of potential key genes and regulatory markers in essential thrombocythemia through integrated bioinformatics analysis and clinical validation," Pharmacogenomics and Personalized Medicine, vol. 14, pp. 767-784, 2021.

[21] Y. Liang, B. Ma, P. Jiang, and H.-M. Yang, "Identification of methylation-regulated differentially expressed genes and related pathways in hepatocellular carcinoma: a study based on tcga database and bioinformatics analysis," Frontiers in oncology, vol. 11, Article ID 636093, 2021.

[22] I. Sierra, J. M. Latorre-Estivalis, L. Traverso et al., "Transcriptomic analysis and molecular docking reveal genes involved in the response of Aedes aegypti larvae to an essential oil extracted from Eucalyptus," PLoS Neglected Tropical Diseases, vol. 15, no. 7, Article ID e0009587, 2021.

[23] Y. Ren, P. Wang, C. Wu, J. Zhang, and C. Niu, "Identification of the metabolites after oral administration of extract of ziziphi spinosae semen to rats or dogs by high-performance liquid chromatography/linear ion trap FTICR hybrid mass spectrometry," Biomedical Chromatography, vol. 27, no. 1, pp. 17-26, 2013.

[24] X. Zhu, X. Liu, K. Pei et al., "Development of an analytical strategy to identify and classify the global chemical constituents of Ziziphi Spinosae Semen by using UHPLC with quadrupole time-of-flight mass spectrometry combined with multiple data-processing approaches," Journal of Separation Science, vol. 41, no. 17, pp. 3389-3396, 2018.

[25] W. Wang, Q. Huang, Y. Chen et al., "The novel FAT4 activator jujuboside A suppresses NSCLC tumorigenesis by activating HIPPO signaling and inhibiting YAP nuclear translocation," Pharmacological Research, vol. 170, Article ID 105723, 2021.
[26] Z. Li, C. Yang, Z. Li, Y. Sun, S. Lin, and Y. Hu, “Application and safety evaluation of an anti-aflatoxigenic chitosan pouch containing turmeric essential oil in the storage of traditional Chinese health food," International Journal of Biological Macromolecules, vol. 183, pp. 1948-1958, 2021.

[27] F. Xu, X. Zhang, J. Wang et al., "Spinosin protects N2a cells from $\mathrm{H}_{2} \mathrm{O}_{2}$-induced neurotoxicity through inactivation of p38MAPK," Journal of Pharmacy and Pharmacology, vol. 72, no. 11, pp. 1607-1614, 2020.

[28] H. Xu, Y. Niu, Y. Sun, M. Xia, D. Lin, and W. Qiao, "Separation and purification of magnoflorine, spinosin, and $6^{\prime \prime \prime}$ feruloyspinosin from Ziziphi Spinosae Semen by high-speed counter-current chromatography," Journal of Separation Science, vol. 44, no. 12, pp. 2391-2398, 2021.

[29] J. Shang, X. Chen, L. Li et al., "Identification of the absorptive constituents and their metabolites in vivo of Ziziphi Spinosae Semen by UPLC-ESI-Q-TOF-MS/MS," Biomedical Chromatography: Biomedical Chromatography, vol. 34, no. 12, Article ID e4965, 2020.

[30] C. Du, Y. Yan, C. Shen, X. Cui, X. Pei, and X. Qin, "Comparative pharmacokinetics of six major compounds in normal and insomnia rats after oral administration of Ziziphi Spinosae Semen aqueous extract," Journal of pharmaceutical analysis, vol. 10, no. 4, pp. 385-395, 2020.

[31] S. Fang, X. Qu, and X. Shu, "Network pharmacology study of Suanzaoren (Semen Ziziphi Spinosae) in treatment of neuropsychiatric diseases," Liaoning Journal of Traditional Chinese Medicine, vol. 47, no. 7, pp. 128-131, 2020.

[32] C. Du, X. Cui, X. Pei, Y. Yan, and X.-M. Qin, "Research progress on Ziziphi Spinosae Semen saponins and its Biological action on nervous system," Chinese Traditional and Herbal Drugs, vol. 50, no. 5, pp. 1258-1268, 2019.

[33] M. Li, F.-X. Zhang, Z.-C. Wei, Z.-T. Li, G.-X. Zhang, and H.-J. Li, "Systematically characterization of in vivo substances of Ziziphi Spinosae Semen in rats by ultra-high-performance liquid chromatography coupled with quadrupole time-offlight tandem mass spectrometry analysis," Journal of Pharmaceutical and Biomedical Analysis, vol. 193, Article ID 113756, 2021.

[34] J. Wu, X. Ye, S. Yang, H. Yu, L. Zhong, and Q. Gong, "Systems pharmacology study of the anti-liver injury mechanism of citri reticulatae pericarpium," Frontiers in Pharmacology, vol. 12, Article ID 618846, 2021.

[35] Y. Xu, X. Li, D. Man, X. Su, and A. Gula, "iTRAQ-based proteomics analysis on insomnia rats treated with Mongolian medical warm acupuncture," Bioscience Reports, vol. 40, no. 5, 2020.

[36] P. Quintin and P. Thomas, "La place des antipsychotiques atypiques dans le traitement des syndromes dépressifs," L'Encéphale, vol. 30, no. 6, pp. 583-589, 2004.

[37] N. K. Y. Tang, "Cognitive behavioural therapy in pain and psychological disorders: towards a hybrid future," Progress in Neuro-Psychopharmacology and Biological Psychiatry, vol. 87, pp. 281-289, 2018.

[38] M. Jansson-Fröjmark, K. Danielsson, A. Markström, and J.-E. Broman, "Developing a cognitive behavioral therapy manual for delayed sleep-wake phase disorder," Cognitive Behaviour Therapy, vol. 45, no. 6, pp. 518-532, 2016.

[39] G. Gartlehner, B. Nussbaumer-Streit, B. N. Gaynes et al., "Second-generation antidepressants for preventing seasonal affective disorder in adults," Cochrane Database of Systematic Reviews, vol. 3, Article ID CD011268, 2019.

[40] J. S. Borchert, B. Wang, M. Ramzanali, A. B. Stein, L. M. Malaiyandi, and K. E. Dineley, "Adverse events due to 
insomnia drugs reported in a regulatory database and online patient reviews: comparative study," Journal of Medical Internet Research, vol. 21, no. 11, Article ID e13371, 2019.

[41] X. S. Fang, J. F. Hao, H. Y. Zhou, L. X. Zhu, J. H. Wang, and F. Q. Song, "Pharmacological studies on the sedative-hypnotic effect of Semen Ziziphi spinosae (Suanzaoren) and Radix et Rhizoma Salviae miltiorrhizae (Danshen) extracts and the synergistic effect of their combinations," Phytomedicine, vol. 17, no. 1, pp. 75-80, 2010.

[42] J.-X. Cao, Q.-Y. Zhang, S.-Y. Cui et al., "Hypnotic effect of jujubosides from semen ziziphi spinosae," Journal of Ethnopharmacology, vol. 130, no. 1, pp. 163-166, 2010.

[43] C.-Y. Ren, J.-X. Rao, X.-X. Zhang, M. Zhang, L. Xia, and G.-H. Chen, "Changed signals of blood adenosine and cytokines are associated with parameters of sleep and/or cognition in the patients with chronic insomnia disorder," Sleep Medicine, vol. 81, pp. 42-51, 2021.

[44] P. Panula, "Histamine receptors, agonists, and antagonists in health and disease," The Human Hypothalamus-Middle and Posterior Region, vol. 180, pp. 377-387, 2021.

[45] T. L. Horvath and X.-B. Gao, "Input organization and plasticity of hypocretin neurons," Cell Metabolism, vol. 1, no. 4, pp. 279-286, 2005.

[46] O. Bruni, L. Ferini-Strambi, E. Giacomoni, and P. Pellegrino, "Herbal remedies and their possible effect on the GABAergic system and sleep," Nutrients, vol. 13, no. 2, 2021.

[47] M. Wimmer, R. Cui, J. Blackwell, and T. Abel, "Cyclic AMP response element-binding protein is required in excitatory neurons in the forebrain to sustain wakefulness," Sleep, vol. 44, no. 6, 2021.

[48] J. P. Coble, M. D. Cassell, D. R. Davis, J. L. Grobe, and C. D. Sigmund, "Activation of the renin-angiotensin system, specifically in the subfornical organ is sufficient to induce fluid intake," American Journal of Physiology-Regulatory, Integrative and Comparative Physiology, vol. 307, no. 4, pp. R376R386, 2014. 